\title{
CARACTERIZAÇÃO ESPACIAL DO AMBIENTE TERMOACÚSTICO E DE ILUMINÂNCIA EM GALPÃO COMERCIAL PARA CRIAÇÃO DE FRANGOS DE CORTE
}

\section{TADAYUKI YANAGI JÚNIOR ${ }^{1}$, ADRIANA G. DO AMARAL ${ }^{2}$, VITOR H. TEIXEIRA ${ }^{3}$, RENATO R. DE LIMA ${ }^{4}$}

RESUMO: A presente pesquisa teve o objetivo de avaliar e espacializar as variáveis termoacústicas e de iluminância no interior de um galpão comercial, com sistema de ventilação convencional e resfriamento evaporativo, destinado à produção de frangos de corte. Os frangos de corte Hybro foram alojados em um galpão subdividido em três regiões (refugos, fêmeas e machos), sendo criados a uma densidade aproximada de 20 aves $\mathrm{m}^{-2}$. $\mathrm{O}$ ambiente produtivo foi avaliado por meio do índice de conforto térmico, nível de ruído e de iluminância. Mapas de distribuição espacial destas variáveis foram gerados para o interior do galpão, usando o método da krigagem. Com base nos resultados, pode-se concluir que as aves, de forma geral, estiveram submetidas a condições ambientais inadequados à criação nos horários mais quentes do dia. Porém, verificou-se o potencial de exploração do sistema de resfriamento evaporativo. A espacialização das variáveis relacionadas ao ambiente produtivo proporciona a obtenção de informações mais detalhadas sobre o sistema estudado.

PALAVRAS-CHAVE: avicultura, ambiente de produção, distribuição espacial.

\section{SPATIAL CHARACTERIZATION OF THERMOACOUSTIC AND LUMINANCE ENVIRONMENT IN COMMERCIAL BROILER CHICKEN HOUSE}

\begin{abstract}
The goal of this study was to evaluate and map the thermo-acoustic and the luminance variables inside of a commercial broiler chicken house equipped with conventional ventilation and misting system. Hybro breed broiler chickens were lodged in three regions (refuse, female and male), at a density of approximately 20 birds $\mathrm{m}^{-2}$. The productive environment was evaluated by the black-globe humidity index, relative humidity, air velocity, noise level and luminance. Spatial distribution maps of these variables were generated inside the broiler house using the kriging method. Based on the results, it was concluded that, in general, broiler chickens were subjected to inadequate environmental conditions during the hottest period of the day; however, the exploration potential for using evaporative cooling systems was verified. The spatial profile of the variables related to the productive environment provide for the attainment of more detailed information about the studied system.
\end{abstract}

KEYWORDS: Aviculture, production environment, spatial distribution.

\section{INTRODUÇÃO}

A importância dos estudos do ambiente na produção avícola tem sido discutida ao longo do tempo, como a influência do ambiente térmico na ingestão de alimentos; no entanto, a interação de diversos fatores ambientais, tais como os fatores térmicos, o nível de ruído e a intensidade de iluminação a que estes animais vêm sendo expostos, não têm sido adequadamente avaliados.

\footnotetext{
${ }^{1}$ Professor Associado, Departamento de Engenharia, Universidade Federal de Lavras, Lavras - MG.

${ }^{2}$ Professora Assistente, Instituto de Ciências Agrárias e Ambientais, Universidade Federal de Mato Grosso - Câmpus Sinop.

${ }^{3}$ Professor Associado, Departamento de Engenharia, Universidade Federal de Lavras, Lavras - MG.

${ }^{4}$ Professor Adjunto II, Departamento de Ciências Exatas, Universidade Federal de Lavras, Lavras - MG.

Recebido pelo Conselho Editorial em: 15-4-2010
}

Aprovado pelo Conselho Editorial em: 17-11-2010 
Assim, para se maximizar a produtividade, é imprescindível aliar um elevado potencial genético do plantel, a uma alimentação com nível nutricional adequado, em ambiente asséptico e ajustado às necessidades das aves. Neste contexto, o conhecimento do ambiente térmico, acústico e de iluminamento no interior do galpão é de vital importância para que os frangos de corte atinjam todo o seu potencial produtivo.

Outro aspecto relevante no estudo do ambiente de produção trata da homogeneidade das variáveis no interior da instalação, que pode ser avaliada por meio da espacialização destas variáveis. Segundo DIGGLE \& RIBEIRO JÚNIOR (2006), a técnica da geoestatística permite descrever espacialmente a variabilidade se a localização espacial variar continuamente na área.

A espacialização das variáveis do ambiente térmico, acústico e de iluminância possibilita entender a relação existente entre estes fatores do ambiente produtivo e sua variação espacial (MIRAGLIOTTA et al., 2006). Outros estudos também têm utilizado a geoestatística como metodologia para avaliar condições de transporte de frangos de corte para o abate (BARBOSA FILHO et al., 2009) e avaliações do ambiente produtivo (DAMASCENO, 2008).

Com base no exposto, a presente pesquisa teve por objetivo a avaliação e a especialização das variáveis termoacústicas e de iluminância em um galpão comercial convencional destinado à produção de frangos de corte.

\section{MATERIAIS E MÉTODOS}

O presente experimento foi conduzido em uma granja comercial de frangos de corte localizada no município de Juiz de Fora - MG, situada na latitude $21^{\circ} 45^{\prime}$ S e longitude $43^{\circ} 21^{\prime} \mathrm{W}$, altitude de $695 \mathrm{~m}$, entre os dias 6 de fevereiro e 4 de março de 2009, do $21^{\circ}$ dia de vida das aves até o $46^{-}$dia de vida. O clima da região é classificado segundo Köeppen como Cwa, com verões quentes e chuvosos, e invernos frios e secos.

O galpão, orientado na direção leste-oeste (Figura 1), possui 2,5 m de pé-direito, cobertura de telhas de cimento-amianto de $4 \mathrm{~mm}$ de espessura, piso de concreto, cama de serragem, cortinas laterais e forro de lona plástica nas cores azul e amarela, respectivamente. Os frangos de corte foram submetidos a um programa de luz contínuo (24L:0E). O galpão foi dividido, por meio de chapas de compensado, em três regiões: região $\mathrm{M}$, região $\mathrm{F}$ e região $\mathrm{R}$, destinadas a machos, fêmeas e refugos, respectivamente, com densidade média de 20,99 fêmeas $\mathrm{m}^{-2}$ e 19,70 machos $\mathrm{m}^{-2}$.

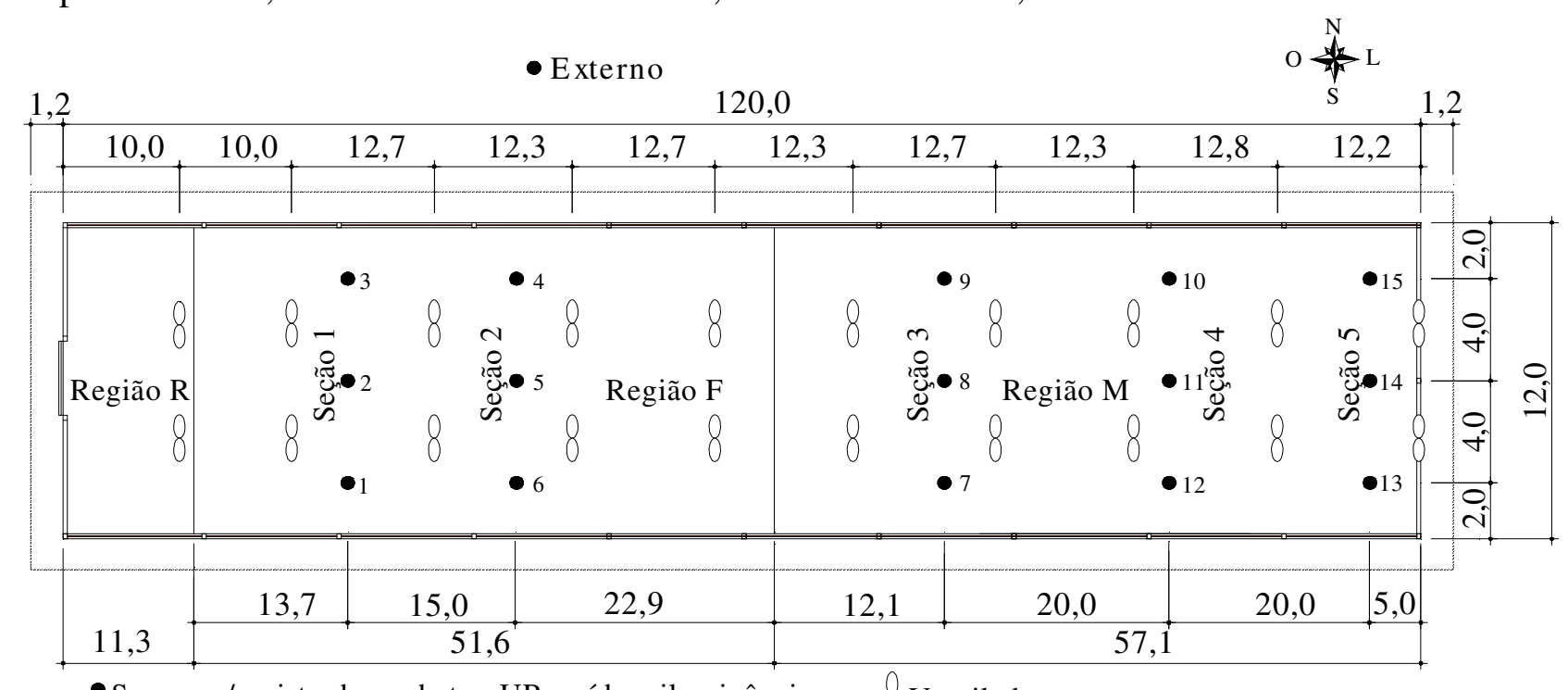

- Sensores/registradores de tar, UR, ruído e iluminância

Ventiladores

FIGURA 1. Esquema do galpão experimental. Unidade de cotagem: metros. Schematic representation of the experimental broiler chicken house. Dimensioning unit: meters. 
O sistema de arrefecimento térmico do ambiente de criação adotado é do tipo convencional, com cortinas laterais abertas continuamente, contando com sistema de ventilação, composto por 20 ventiladores (112 cv de potência cada e vazão de $240 \mathrm{~m}^{3} \mathrm{~min}^{-1}$ ), associado a um sistema de nebulização (Figura 1). O sistema de nebulização é composto por duas linhas equipadas com 10 nebulizadores $\left(7 \pm 1,8 \mathrm{~L} \mathrm{~h}^{-1}\right)$ cada.

As variáveis que compõem o ambiente térmico, temperatura do ar $\left(\mathrm{t}_{\mathrm{ar}}\right)$, temperatura de ponto de orvalho $\left(\mathrm{t}_{\mathrm{po}}\right)$, umidade relativa (UR) e temperatura de globo negro $\left(\mathrm{t}_{\mathrm{gn}}\right)$, assim como o nível de ruído e a iluminância, foram coletadas simultaneamente por meio de sensores/registradores, a cada 10 minutos, no período das $8 \mathrm{~h}$ às $18 \mathrm{~h}$, em quinze pontos alocados no interior do galpão, a $0,30 \mathrm{~m}$ de altura em relação ao piso, e em um ponto no ambiente externo (Figura 1). Os sensores/registradores de $\mathrm{t}_{\mathrm{ar}}$ e UR (Hobo $\mathrm{H} 08$ Pro, precisão de $\pm 0,2^{\circ} \mathrm{C} \mathrm{e} \pm 3 \%$, e; Instrutherm HT500, precisão $\pm 1{ }^{\circ} \mathrm{C}$ e $\pm 3 \%$, respectivamente ), $t_{\text {gn }}$ (Hobo $\mathrm{H} 08$, precisão de $0,7^{\circ} \mathrm{C}$ à $21,1^{\circ} \mathrm{C}$ e Instrutherm TH-060, precisão de $0,1{ }^{\circ} \mathrm{C}$ a $0,8{ }^{\circ} \mathrm{C}$ para temperaturas de $0{ }^{\circ} \mathrm{C}$ a $200{ }^{\circ} \mathrm{C}$ ) e iluminância (Instrutherm, modelo LDR-380 precisão de $\pm 3 \%$, ), e os decibelímetros (Instrutherm, modelo DEC480 , precisão de $\pm 1,5 \mathrm{~dB}$ ) foram mantidos em gaiolas de arame a fim de que os animais não os danificassem. A velocidade do ar $\left(\mathrm{V}_{\mathrm{ar}}\right)$ foi medida de modo pontual a 0,3 m de altura, por meio de um anemômetro digital de hélices (Kestrel ${ }^{\circledR} 4000$, precisão de $\pm 3 \%$ da leitura), próxima aos globos negros.

Para a velocidade do ar $\left(\mathrm{V}_{\mathrm{ar}}\right)$, foi realizada uma divisão virtual do galpão em malhas com pontos espaçados em toda a extensão da instalação, sendo 2 metros e 5 metros entre si, no sentido da largura e do comprimento, respectivamente (Figura 2), totalizando 350 pontos, sendo que, em cada ponto, foram realizadas três repetições. As medições de $\mathrm{V}_{\mathrm{ar}}$ foram obtidas de modo pontual, a $0,3 \mathrm{~m}$ de altura, correspondendo à altura das aves. As velocidades do ar foram medidas em horários aleatórios, durante os dias de coleta, com os ventiladores ligados. O tempo de medição para a variável $\mathrm{V}_{\mathrm{ar}}$ foi de $30 \mathrm{~min}$.

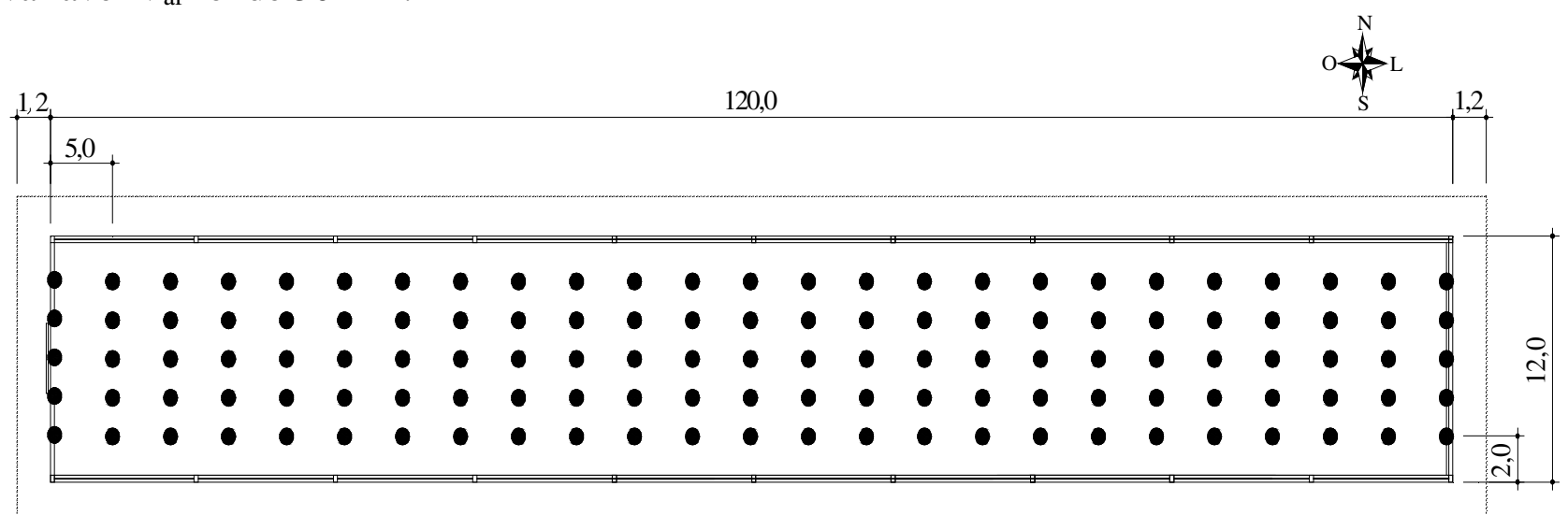

FIGURA 2. Pontos de coleta da $\mathrm{V}_{\text {ar }}$ no interior de um galpão de frangos de corte. Unidade de cotagem: metros. Measurement points of the $\mathbf{V}_{\text {ar }}$ inside of the broiler chicken house. Dimensioning unit: meters.

Os valores de ITGU foram calculados a cada $10 \mathrm{~min}$, em função dos dados de $t_{\text {gn }}$ e $t_{p o}$ medidos, por meio da eq.(1) (BUFFINGTON et al., 1981):

$$
\mathrm{ITGU}=\mathrm{t}_{\mathrm{gn}}+0,36 \mathrm{t}_{\mathrm{po}}-330,08
$$

em que,

$\mathrm{t}_{\mathrm{gn}}$ - temperatura de globo negro, $\mathrm{K}, \mathrm{e}$

$\mathrm{t}_{\mathrm{po}}$ - temperatura de ponto de orvalho, $\mathrm{K}$. 
O ambiente de produção foi analisado e descrito ao longo do período avaliado, por meio da estatística descritiva, uma vez que o período experimental compreendeu todo o ciclo produtivo, sendo coletada uma grande quantidade de informações sobre o ambiente. Assim, foram obtidas as distribuições de frequências e construídos histogramas referentes às avaliações em todo o período experimental, fixando os horários de $8 ; 10 ; 12 ; 14 ; 16$ e $18 \mathrm{~h}$. Além disso, medidas de posição e de dispersão, como a média e o desvio-padrão, valores máximos e mínimos, foram determinadas para cada uma das variáveis avaliadas, ou seja, ITGU, UR, $\mathrm{V}_{\text {ar }}$, ruído e iluminância.

Para descrever as variáveis termoacústicas, UR, $\mathrm{V}_{\mathrm{ar}}$, nível de ruído, índice de conforto e de iluminamento, utilizou-se a geoestatística, técnica baseada na teoria das variáveis regionalizadas (GOOVAERTS, 2001). A dependência espacial foi analisada por meio de ajustes de semivariogramas (VIEIRA, 2000), com base na pressuposição de estacionariedade (hipótese intrínseca), a qual é estimada pela eq.(2):

$$
\widehat{\gamma}(h)=\frac{1}{2 N(h)} \sum_{i=1}^{N(h)}\left[z\left(x_{i}\right)-z\left(x_{1}+h\right]^{z}\right.
$$

em que,

$\mathrm{N}(\mathrm{h})$ - número de pares experimentais de dados separados por uma distância h;

$\mathrm{Z}\left(\mathrm{x}_{\mathrm{i}}\right)$ - valor determinado em cada ponto de leitura, e,

$\mathrm{Z}\left(\mathrm{x}_{\mathrm{i}}+\mathrm{h}\right)$ - valor medido num ponto, mais uma distância $\mathrm{h}$.

Dessa forma, foram estimados os coeficientes do modelo teórico para o semivariograma (efeito pepita, $\mathrm{C}_{0}$; patamar, $\mathrm{C}_{0}+\mathrm{C}_{1}$; alcance, A). Testaram-se semivariagramas do tipo esférico, exponencial, linear, linear com patamar e gaussiano (SOUZA et al., 2004), ajustados por meio do programa GS+. A escolha dos modelos matemáticos foi realizada, observando-se o coeficiente de correlação obtido pela técnica de validação cruzada. Essa técnica consiste em retirar, individualmente, cada ponto medido da área estudada; o seu valor é estimado, via krigagem, como se ele nunca existisse.

Posteriormente, através do programa Surfer, tais modelos foram utilizados na obtenção de mapas de krigagem, método de inferência espacial que estima dados em pontos não amostrados a partir dos pontos amostrados.

Os mapas de krigagem das variáveis ITGU, UR e iluminância foram obtidos para diferentes horários ao longo do dia, ou seja: $8 ; 10 ; 12 ; 14 ; 16$ e 18h. Já para as variáveis, nível de ruído e a $\mathrm{V}_{\mathrm{ar}}$, tomou-se como base os valores médios observados em todo o período experimental, gerando-se um único mapa.

Os dados coletados para cada variável foram espacializados e distribuídos em classes. Para as variáveis térmicas ITGU, UR e $\mathrm{V}_{\mathrm{ar}}$, as classes foram definidas, tomando-se como base a classificação de conforto térmico proposta por MEDEIROS et al. (2005), em que valores de ITGU inferiores a 69 indicam condição de estresse por frio, entre 69 e 77, condição de conforto e acima de 77 estresse por calor. Estas classes foram subdividas ao meio, permitindo, desta maneira, a caracterização mais detalhada do ambiente produtivo no interior do galpão. Na construção das classes para as variáveis nível de ruído e a iluminância, foram utilizados os valores observados experimentalmente, conforme Tabela 1.

\section{RESULTADOS E DISCUSSÃO}

Na Tabela 1, estão apresentados os valores mínimos, máximos, médios e os desvios-padrão estimados para as variáveis ITGU, UR, $\mathrm{V}_{\text {ar }}$, ruído e iluminância. Os valores de ITGU foram similares para todas as seções longitudinais do galpão (norte, centro e sul), bem como, para todo o galpão (ITGU geral). Estes valores encontram-se acima do limite de conforto indicado para frangos de corte adultos (ITGU>77) (MEDEIROS et al., 2005). Observa-se também que diferiu do ambiente 
externo, já que este atingiu valores medianos da ordem de 91,7 , portanto a instalação amortizou o ITGU em aproximadamente $13 \%$.

Em média, os valores de UR mantiveram-se no intervalo considerado como de conforto, ou seja, entre 50 e $80 \%$ (MEDEIROS et al., 2005). Entretanto, valores mínimos e máximos indicaram que, em alguns períodos do dia e em certas partes do galpão, foram observados níveis de UR inferiores e superiores ao intervalo de conforto (Tabela 1). Com relação ao ambiente externo, observa-se que a UR apresentou valores medianos inferiores ao da instalação.

A $V_{a r}$ média no interior do galpão foi de $0,9 \mathrm{~m} \mathrm{~s}^{-1}$, inferior ao intervalo recomendado para frangos de corte adultos, que é de 1,5 a 2,5 $\mathrm{m} \mathrm{s}^{-1}$ (MEDEIROS et al., 2005).

Por sua vez, os valores medianos de ruído foram de 74,4 dBA e, externamente ao galpão, de 69,3 dBA. Para a iluminância, a mediana foi de 590 lux $\mathrm{m}^{-2}$, entretanto valores extremos foram observados no interior do galpão. Enquanto o valor de ruído está próximo ao encontrado por Miragliotta et al. (2006), que foi de 69 dBA, a iluminância média foi 2,65 vezes maior. A diferença obtida neste último resultado deve-se ao fato de estes autores terem avaliado um galpão com ventilação em modo túnel, que se caracteriza por manter as cortinas fechadas durante o funcionamento em modo túnel, atenuando a contribuição da luz externa, advinda do sol.

TABELA 1. Valores mínimos, máximos, médios, medianos e desvios-padrão das variáveis termoacústica e de iluminância medidas no interior de um galpão convencional para criação comercial de frangos de corte. Minimum, maximum, average, median and standard deviations values of the thermo-acoustic and luminance variables measured inside a conventional commercial broiler chicken house.

\begin{tabular}{cccccc}
\hline Variáveis & Mínimo & Máximo & Média & Mediana & Desvio-Padrão \\
\hline ITGU $_{\text {Geral }}$ & 65,3 & 94,4 & 79,1 & 79,4 & 3,2 \\
ITGU Face norte $_{\text {ITGU }}$ Centro & 65,9 & 94,4 & 79,5 & 79,9 & 3,2 \\
ITGU $_{\text {Face sul }}$ & 69,2 & 93,8 & 79,5 & 79,9 & 3,1 \\
ITGU $_{\text {Externo }}$ & 65,3 & 87,7 & 79,4 & 79,8 & 3,0 \\
UR (\%) & 67,7 & 100,3 & 89,6 & 91,7 & 11,3 \\
UR $_{\text {Externo }}(\%)$ & 38 & 100 & 67,6 & 66,2 & 12,6 \\
V $_{\text {ar }}\left(\right.$ m s $\left.^{-1}\right)$ & 24,2 & 99,3 & 68,9 & 60 & 41,6 \\
Ruído (dBA) $_{\text {Ruído }}$ & 0,0 & 2,6 & 0,9 & 0,8 & 0,5 \\
Iluminância (dBA) & 35 & 100 & 72,1 & 74,4 & 7,3 \\
\hline
\end{tabular}

ITGU - índice de temperatura do globo negro e umidade; UR - umidade relativa (\%); $\mathrm{V}_{\mathrm{ar}}$ - velocidade do ar ( $\mathrm{m} \mathrm{s}^{-1}$ ); Ruído - nível de ruído (dBA); Iluminância $\left(l u x \mathrm{~m}^{-2}\right)$.

A decisão pelo modelo de melhor ajuste ao atributo foi feita com base na validação cruzada considerando os critérios observados por MCBRATNEY \& WEBSTER (1986), CRESSIE (1993) e VIEIRA (2000), que trata da condição de não tendenciosidade, valor populacional para o erro médio reduzido (EMR) igual a zero e desvio-padrão do erro reduzido $\left(\mathrm{S}_{\mathrm{ER}}\right)$ igual a um. Assim, o modelo de melhor ajuste foi aquele que teve o ER mais próximo de zero e o $\mathrm{S}_{\mathrm{ER}}$ mais próximo de um, sendo observada a maior presença do modelo esférico, concordando com os resultados encontrados por FARIA et al. (2008) ao avaliar ambiente produtivo para bovinos de leite criados em confinamento. Também ocorreu o efeito pepita puro (EPP) para o atributo luminosidade nos horários de observação de 10h, 12h e 14h, que indicam a ausência total de dependência espacial, ou seja, a dependência espacial, se existir, será manifestada a distância ou tempo menor do que o menor espaçamento entre amostras. 
TABELA 2. Distribuição de frequência, em percentagem, de cada classe para os atributos índice de temperatura de globo negro e umidade (ITGU), umidade relativa (UR,\%), luminosidade (lux) e nível de ruído (dBA), nos diferentes horários de observação. Frequency distribution, in percentage, of each class for the attributes Black globe humidity index (BGHI), relative humidity (RH, \%), luminance (lux) and noise level (dBA) in different hours of observation.

\begin{tabular}{|c|c|c|c|c|c|c|c|c|}
\hline \multicolumn{9}{|c|}{ Percentagem de classe } \\
\hline Tempo(h) & Classe & ITGU & Classe & UR & Classe & Luminosidade & Classe & Ruído \\
\hline \multirow{3}{*}{8} & $67-69$ & 0 & $10-50$ & 0 & $0-500$ & 33 & $35-50$ & 1 \\
\hline & $69-77$ & 100 & $50-80$ & 20 & $500-750$ & 53 & $50-70$ & 54 \\
\hline & $77-81$ & 0 & $80-100$ & 80 & $>1000$ & 13 & $70-100$ & 45 \\
\hline \multirow{3}{*}{10} & $67-69$ & 0 & $10-50$ & 0 & $0-500$ & 13 & $35-50$ & 1 \\
\hline & $69-77$ & 100 & $50-80$ & 100 & $500-750$ & 20 & $50-70$ & 27 \\
\hline & $77-81$ & 0 & $80-100$ & 0 & $>1000$ & 67 & $70-100$ & 72 \\
\hline \multirow{3}{*}{12} & $67-69$ & 0 & $10-50$ & 0 & $0-500$ & 7 & $35-50$ & 0 \\
\hline & $69-77$ & 7 & $50-80$ & 100 & $500-750$ & 27 & $50-70$ & 8 \\
\hline & $77-81$ & 93 & $80-100$ & 0 & $>1000$ & 67 & $70-100$ & 92 \\
\hline \multirow{3}{*}{14} & $67-69$ & 0 & $10-50$ & 0 & $0-500$ & 20 & $35-50$ & 0 \\
\hline & $69-77$ & 7 & $50-80$ & 100 & $500-750$ & 27 & $50-70$ & 14 \\
\hline & $77-81$ & 93 & 80-100 & 0 & $>1000$ & 53 & $70-100$ & 86 \\
\hline \multirow{3}{*}{16} & $67-69$ & 0 & $10-50$ & 0 & $0-500$ & 33 & $35-50$ & 1 \\
\hline & $69-77$ & 87 & $50-80$ & 100 & $500-750$ & 67 & $50-70$ & 21 \\
\hline & $77-81$ & 13 & 80-100 & 0 & $>1000$ & 0 & $70-100$ & 78 \\
\hline \multirow{3}{*}{18} & $67-69$ & 0 & $10-50$ & 0 & $0-500$ & 100 & $35-50$ & 1 \\
\hline & $69-77$ & 100 & $50-80$ & 100 & $500-750$ & 0 & $50-70$ & 41 \\
\hline & $77-81$ & 0 & 80-100 & 0 & $>1000$ & 0 & $70-100$ & 58 \\
\hline
\end{tabular}

TABELA 3. Modelos e parâmetros estimados dos semivariogramas experimentais para os atributos, índice de temperatura de globo negro e umidade (ITGU), umidade relativa (UR, \%) e luminosidade (lux), nos diferentes horários de observação, e para a velocidade do ar $\left(\mathrm{V}_{\mathrm{ar}}, \mathrm{m} \mathrm{s}^{-1}\right)$ e nível de ruído (dBA). Models and parameters estimated of the experimental semi-variograms for the attributes, black globe humidity index (BGHI), relative humidity (RH, \%) and luminance (lux) throughout the different hours of observation, and for air velocity $\left(\mathrm{V}_{\text {air }}, \mathrm{m} \mathrm{s}^{-1}\right)$ and noise level (dBA).

\begin{tabular}{|c|c|c|c|c|c|c|c|}
\hline $\begin{array}{l}\text { Tempo } \\
\text { (h) }\end{array}$ & Atributos & Modelo & $\begin{array}{c}\text { Efeito pepita } \\
\left(\mathrm{C}_{0}\right)\end{array}$ & $\begin{array}{l}\text { Patamar } \\
\left(\mathrm{C}_{0}+\mathrm{C}_{1}\right)\end{array}$ & Alcance (A) & ${ }^{1} \mathrm{IDE} \mathrm{C}_{0} / \mathrm{C}_{0}+\mathrm{C}_{1}$ & ${ }^{2} \mathrm{VC}$ \\
\hline \multirow[t]{2}{*}{----- } & $\mathrm{V}_{\mathrm{ar}}$ & Exponencial & 0,01 & 0,12 & 3,84 & 0,88 & 0,77 \\
\hline & Ruído & Exponencial & 0,02 & 3,13 & 6,54 & 0,99 & 1,24 \\
\hline \multirow{3}{*}{8} & ITGU & Linear & 20,34 & 44,22 & 40,26 & 0,54 & 0,00 \\
\hline & UR & Linear & 34,16 & 34,16 & 40,26 & 0,00 & 0,01 \\
\hline & Luminosidade & Esférico & $14.000,00$ & $84.500,00$ & 7,09 & 0,83 & $-0,34$ \\
\hline \multirow{3}{*}{10} & ITGU & Esférico & 0,191 & 2,479 & 8,04 & 0,92 & 0,20 \\
\hline & UR & Linear & 7,53 & 9,21 & 40,26 & 0,18 & 0,02 \\
\hline & Luminosidade & $\mathrm{EPP}^{3}$ & - & - & - & - & - \\
\hline \multirow{3}{*}{12} & ITGU & Linear & 3,58 & 3,76 & 40,26 & 0,05 & 0,00 \\
\hline & UR & Esférico & 0,16 & 3,14 & 6,21 & 0,01 & 0,5 \\
\hline & Luminosidade & $\mathrm{EPP}^{3}$ & - & - & - & - & - \\
\hline \multirow{3}{*}{14} & ITGU & Linear & 4,50 & 5,02 & 40,26 & 0,10 & 0,00 \\
\hline & UR & Gaussiano & 1,84 & 3,98 & 13,94 & 0,54 & 0,35 \\
\hline & Luminosidade & $\mathrm{EPP}^{3}$ & - & - & - & - & - \\
\hline \multirow{3}{*}{16} & ITGU & Esférico & 0,49 & 4,90 & 8,10 & 0,90 & 0,04 \\
\hline & UR & Esférico & 10,7 & 110,80 & 4,88 & 0,90 & $-9,35$ \\
\hline & Luminosidade & Esférico & $2.250,00$ & $32.520,00$ & 8,24 & 0,93 & $-0,04$ \\
\hline \multirow{3}{*}{18} & ITGU & Esférico & 0,21 & 4,05 & 8,14 & 0,98 & 1,11 \\
\hline & UR & Linear & 6,82 & 8,04 & 40,26 & 0,15 & 0,01 \\
\hline & Luminosidade & Esférico & 1,00 & $1.312,00$ & 4,00 & 0,99 & $-12,81$ \\
\hline
\end{tabular}

${ }^{1} \mathrm{C}_{0} /\left(\mathrm{C}_{0}+\mathrm{C}_{1}\right)$ - índice de dependência espacial; ${ }^{2} \mathrm{VC}$ - validação cruzada, ${ }^{3} \mathrm{EPP}$ - efeito pepita puro. 
A distribuição de percentagem de classe pode ser vista através da Tabela 2. Verificou-se que, nos horários de $8 ; 10$ e 18h, todo o galpão apresentava-se em condições de conforto térmico (Figura 3A, B e F), caracterizado por valores de ITGU entre 69 e 77 (MEDEIROS et al., 2005). Entretanto, para as 12 e 14h, aproximadamente 93\% do galpão estava submetido a condições de estresse térmico, ou seja, valores de ITGU maiores que 77 (MEDEIROS et al., 2005). Isto pode ser visualizado pela diferenciação de coloração em pontos ao longo do galpão (Figura 3C e D).

O limite de dependência espacial é estabelecido pelo alcance (A), isto é, nas distâncias iguais ou menores que o alcance, entende-se que os valores vizinhos do atributo estão espacialmente correlacionados e podem ser utilizados para se estimarem valores em qualquer ponto entre eles.

O maior alcance obtido foi de 40,26 m para ITGU às $8 \mathrm{~h}, 12 \mathrm{~h}$ e $14 \mathrm{~h}$, e, para UR, às $8 \mathrm{~h}, 10 \mathrm{~h}$ e 16h; o alcance reflete o grau de homogeneização entre as amostras, ou seja, quanto maior for o alcance, maior será a homogeneidade entre as amostras, demonstrando, assim, que estes atributos demonstram ser mais homogêneos quando comparados aos demais.

Segundo CAMBARDELLA et al. (1994), pelo índice de dependência espacial $\left(\mathrm{C}_{0} /\left(\mathrm{C}_{0}+\mathrm{C}_{1}\right)\right)$, os atributos podem ser classificados quanto ao grau de dependência (Tabela 3). Assim, observa-se que as variáveis $\mathrm{V}_{\mathrm{ar}}$, Ruído, ITGU às 10h, 16h e 18h, UR às 16h, e Luminosidade nos horários de 8; 10; 16 e 18 apresentam fraca dependência espacial, já que os valores encontrados de IDE são superiores a $75 \%$.

Para os atributos ITGU às $12 \mathrm{~h}$ e $14 \mathrm{~h}$ e UR às $8 \mathrm{~h}, 10 \mathrm{~h}, 12 \mathrm{~h}$ e $18 \mathrm{~h}$, ocorreu uma forte dependência, pois os valores de IDE foram inferiores a 25\%. Esses resultados estão de acordo com os obtidos por MILES et al. (2008) e FARIA et al. (2008), que, ao avaliarem ambientes produtivos para frangos de corte e bovinos de leite, encontraram dependência espacial para os atributos climáticos. A moderada dependência ocorre quando o IDE está entre 25 e $75 \%$, neste caso, o ITGU às $8 \mathrm{~h}$ e UR às $14 \mathrm{~h}$ encontram-se nesta classificação. Para o ITGU às $8 \mathrm{~h}$ e UR às $14 \mathrm{~h}$, verificou-se moderada dependência, pois o IDE situou-se entre 25 e 75\% (CAMBARDELLA et al., 1994).

Apesar de alguns resultados indicarem não haver dependência espacial, optou-se pela geração dos mapas a fim de se visualizar melhor a condição ambiental de produção, na qual se observa que, em alguns horários, os animais estavam submetidos a condições ambientais homogêneas.

Nos horários mais quentes do dia, entre 12 e 16h, e em especial próximo às 14h, verificou-se que, apesar de grande parte do galpão encontrar-se em desconforto térmico (Figuras 3C e 3D), existe potencial para se usar o resfriamento evaporativo, uma vez que a UR se encontra entre 50 e $70 \%$ (Figuras 4C e 4D), que, segundo a literatura, é a adequada para se obter a máxima produtividade de frangos de corte (MEDEIROS et al., 2005). Dessa forma, poder-se-á reduzir os valores de ITGU a níveis mais adequados à produção de frangos de corte.

O comportamento crescente e decrescente do ITGU e da UR ao longo do dia, devido à variação diurna da temperatura do ar, também foi relatado por outros autores (SANTOS et al., 2005; FURTADO et al., 2006; ABREU et al., 2007; BIAGGIONI et al., 2008; e DAMASCENO, 2008). 
A.

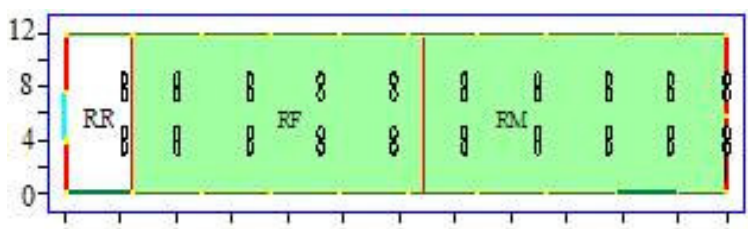

B.

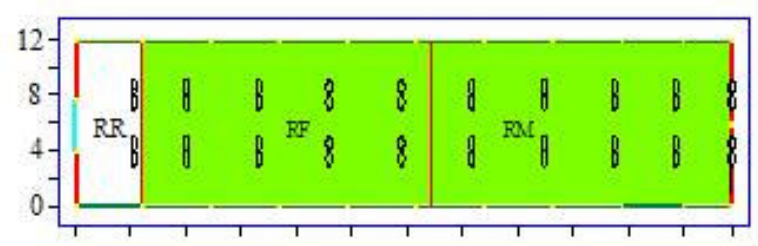

c

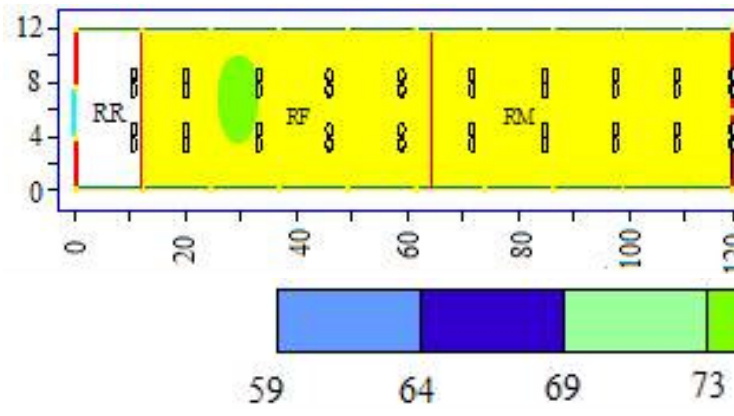

D.

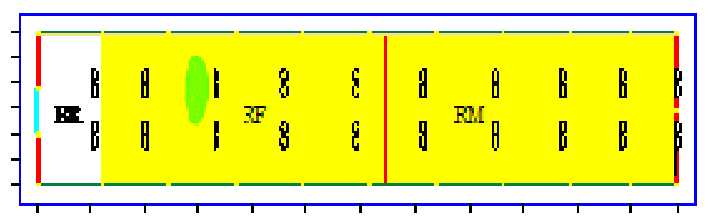

E.

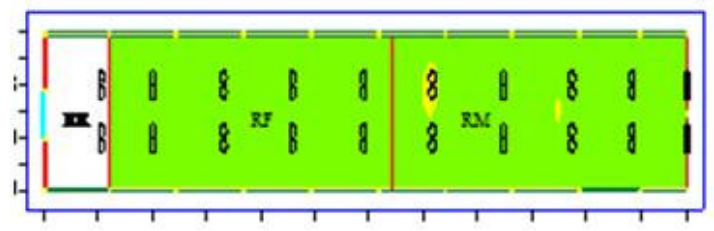

F.

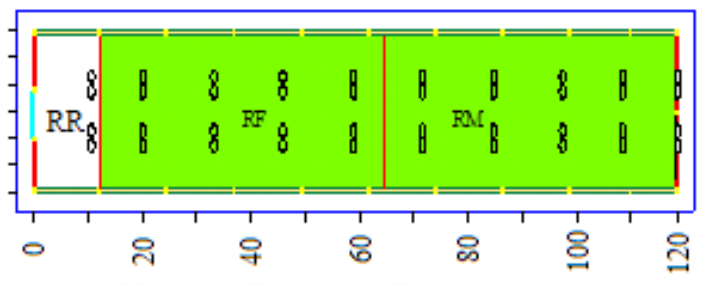

FIGURA 3. Distribuição espacial da variável ITGU no interior de um galpão comercial para produção de frangos de corte, em diferentes horários ao longo do dia: A) 8h; B) 10h; C) $12 \mathrm{~h}$; D) $14 \mathrm{~h}$; E) $16 \mathrm{~h}$, e F) $18 \mathrm{~h}$. Spatial distribution of the BGHI variable inside of a commercial broilers chicken house at different times throughout the day, A) 8:00, B) 10:00, C) 12:00, D) 14:00, E) 16:00, and F) 18:00.

A.

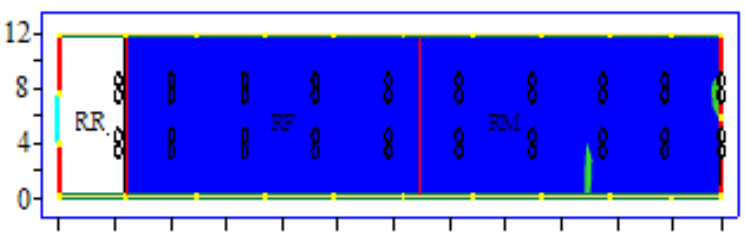

B.

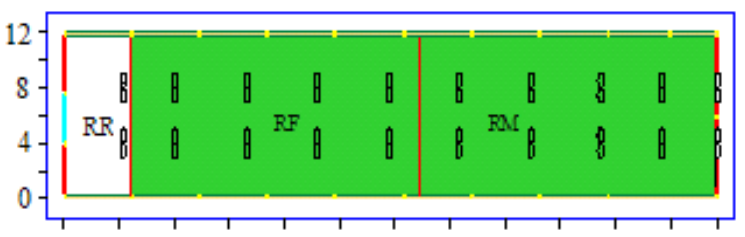

C.

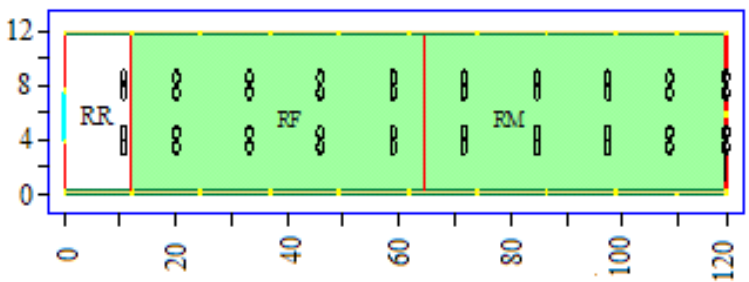

D.

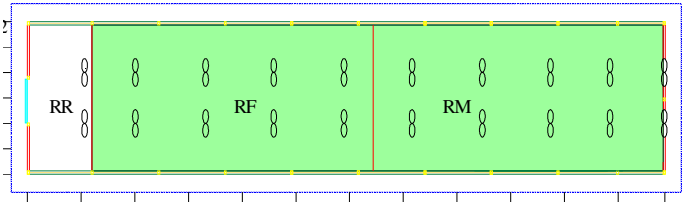

E.

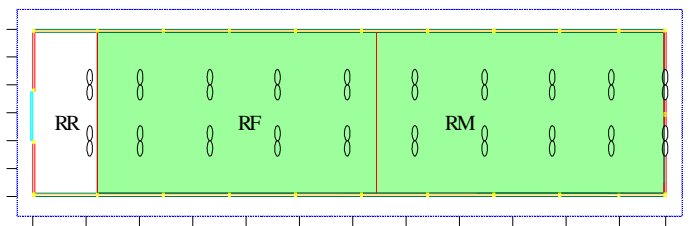

F.

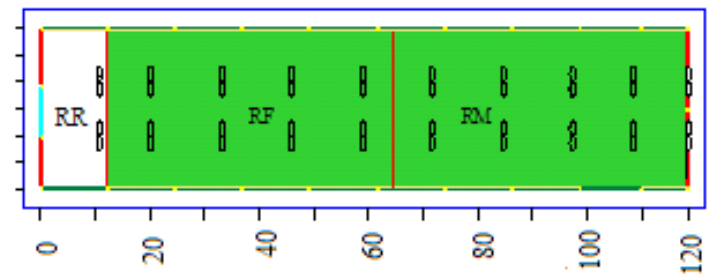

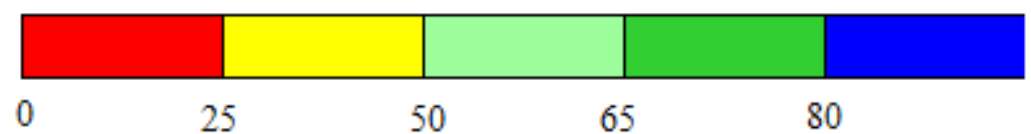

FIGURA 4. Distribuição espacial da UR no interior de um galpão comercial para produção de frangos de corte, em diferentes horários ao longo do dia: A) 8h; B) 10h; C) 12h; D) 14h; E) 16h, e F) 18h. Spatial distribution of $\mathbf{R H}$ inside of a commercial broiler chicken house at different times throughout the day, A) 8:00, B) 10:00, C) 12:00, D) 14:00, E) 16:00, and F) 18:00. 
A distribuição de frequência de $V_{a r}$ mostra que a maior parte das observações $(80,5 \%)$ apresenta valores menores que $1,5 \mathrm{~m} \mathrm{~s}^{-1}$, e apenas $19,5 \%$ dos dados encontram-se dentro da faixa considerada como a que proporciona máxima produtividade aos frangos de corte (Figura 5A), ou seja, $V_{\text {ar }}$ de 1,5 a 2,5 m s$~^{-1}$ (MEDEIROS et al., 2005) (Figura 5A). Pela espacialização dos valores médios de $\mathrm{V}_{\mathrm{ar}}$ (Figura $5 \mathrm{~B}$ ), os menores valores ocorreram próximos às cabeceiras dos galpões, devido à influência das paredes.

A.

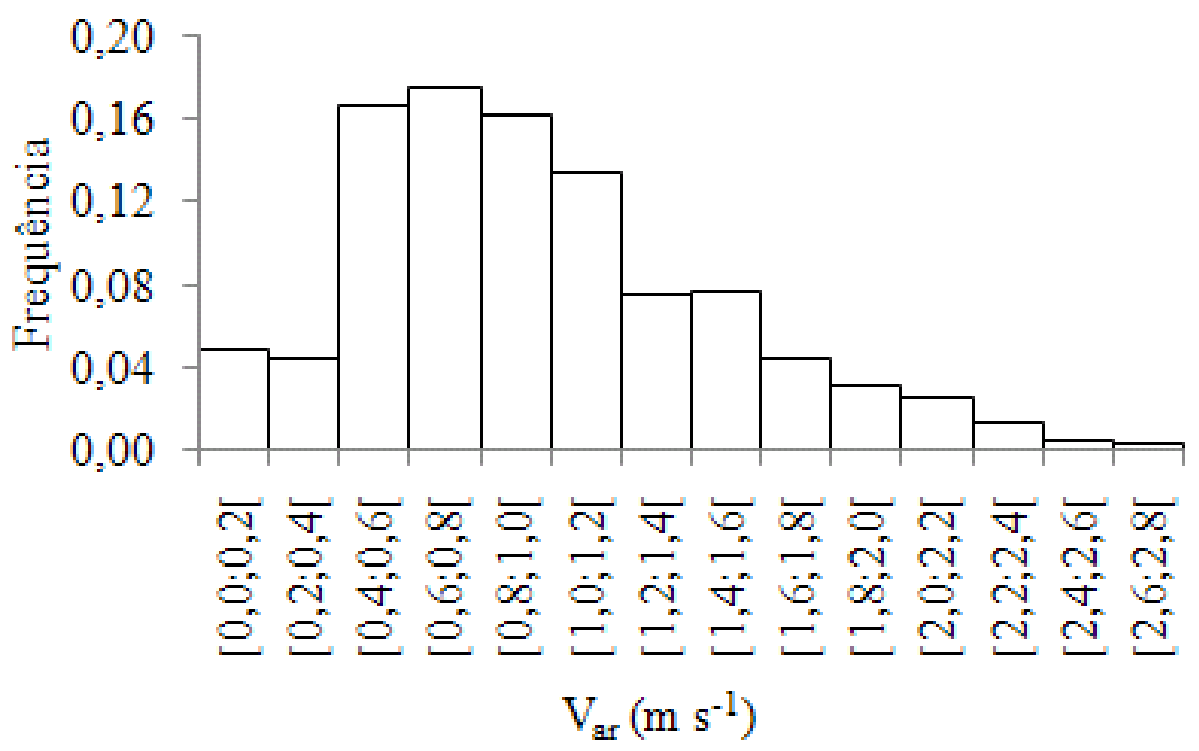

B.

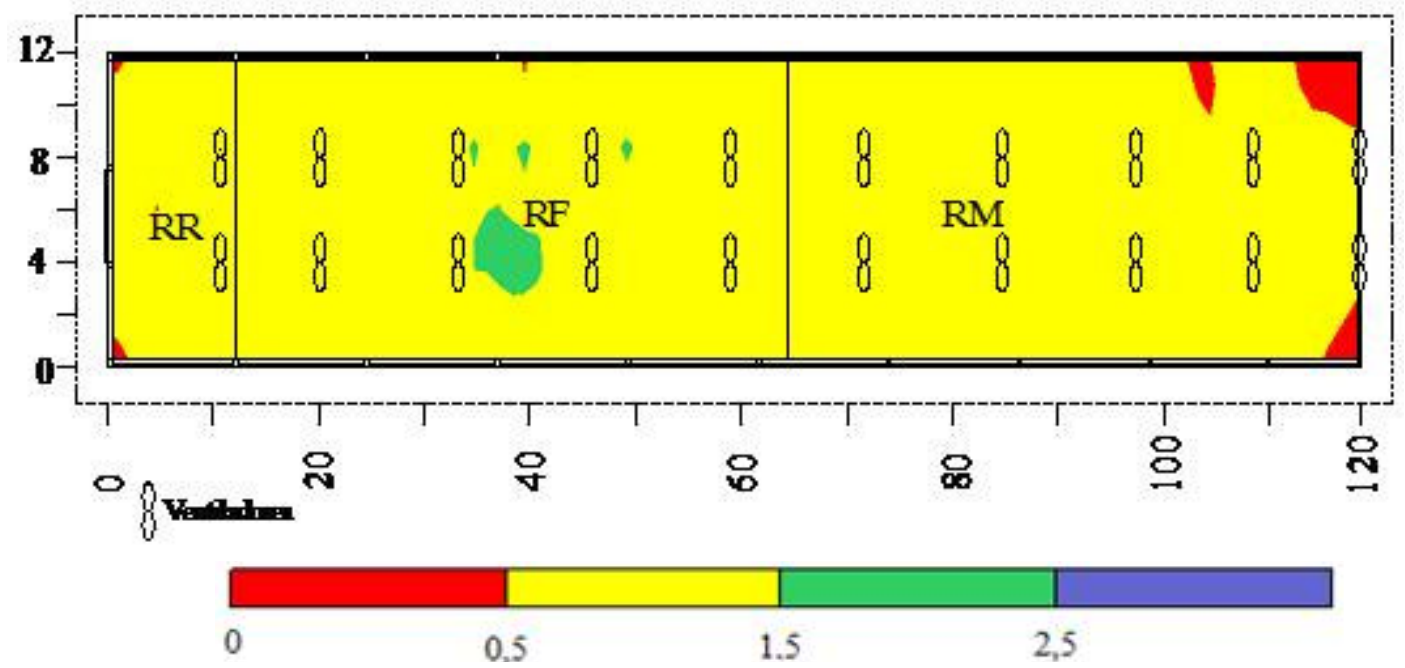

FIGURA 5. Histograma (A) e distribuição espacial da $\mathrm{V}_{\text {ar }}$ no interior de um galpão comercial para produção de frangos de corte (B). Histogram (A) and spatial distribution of $\mathbf{V}_{\mathrm{ar}}$ inside of commercial broilers chicken house $(B)$.

No geral, observa-se que as maiores frequências dos níveis de ruído concentram-se entre 70 e 80 dBA, o que pode ser visualizado espacialmente na Figura 6. Ressalta-se que os maiores valores de ruído foram observados próximo aos ventiladores.

Basicamente, estes ruídos são oriundos dos próprios animais, dos ventiladores e do sistema de arraçoamento, sendo que, eventualmente, observou-se a contribuição de ruídos decorrentes do caminhamento do funcionário da granja movimentando-se no interior do galpão, bem como do tráfego de veículos e máquinas agrícolas próximo ao galpão. 


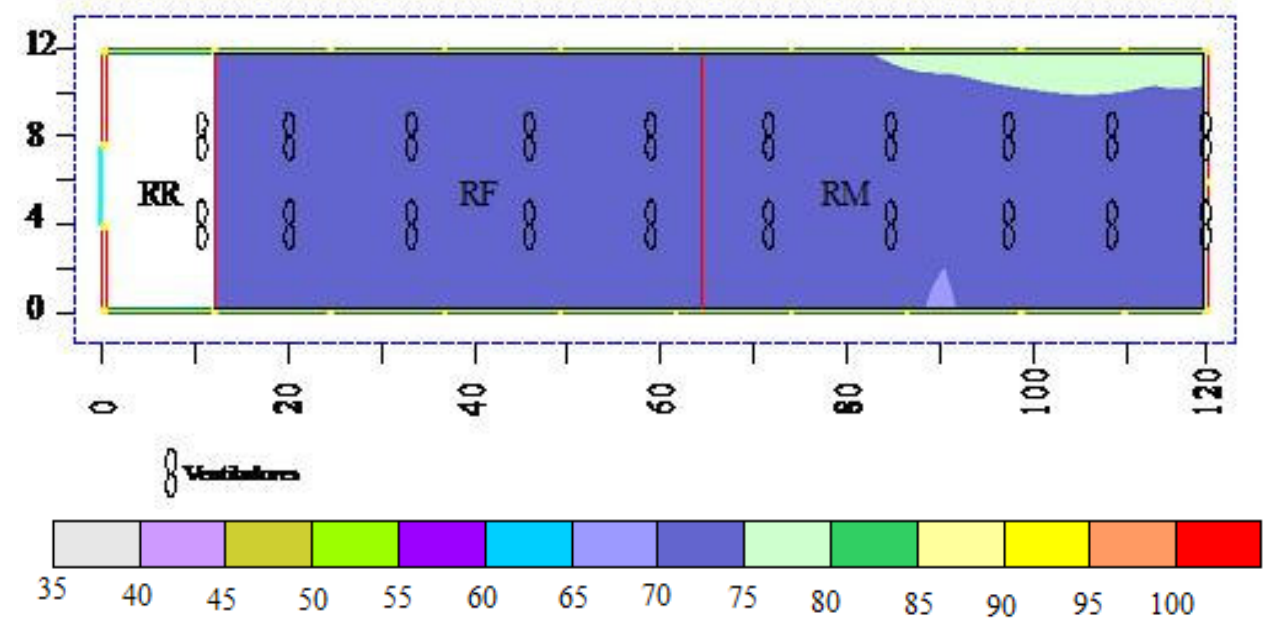

FIGURA 6. Distribuição espacial do nível de ruído (dBA) no interior de um galpão comercial para produção de frangos de corte. Spatial distribution of the noise level (dBA) inside of a commercial broiler chicken house.

No geral, observa-se, que durante praticamente todo o período diurno, a iluminância foi muito superior ao valor recomendado para frangos de corte, que é de 5 lux m $\mathrm{m}^{-2}$ (DOWNS et al., 2006), concentrando-se entre 500 e 750 lux m$^{-2}$ (Figura 7). Ademais, observou-se que, ao longo do dia, analisando os mapas de espacialização, que o centro do galpão, ao nível das aves, recebia menor iluminação. Entretanto, deve-se ressaltar que, em condições normais, as aves estão sujeitas a variação cíclica de luz durante o dia; entretanto, durante a noite, pode-se usar o valor recomendado por DOWNS et al. (2006), visando à redução dos custos fixos e variáveis despendidos com o sistema de iluminação.

A.
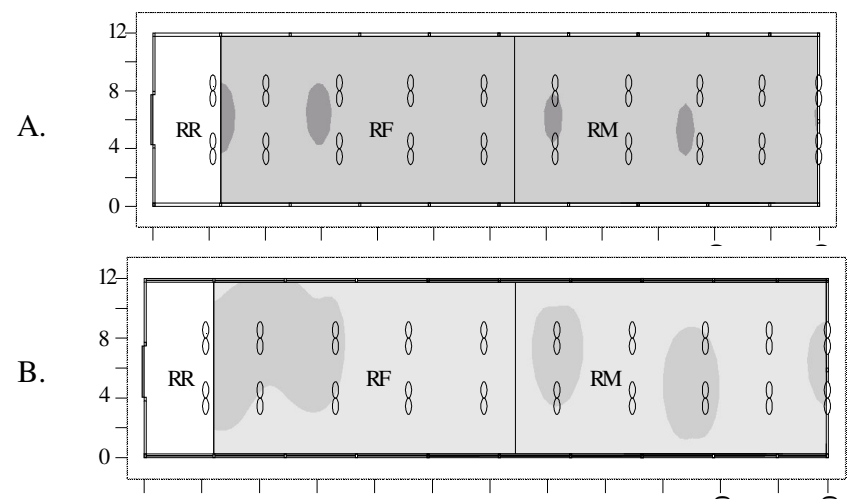

C.
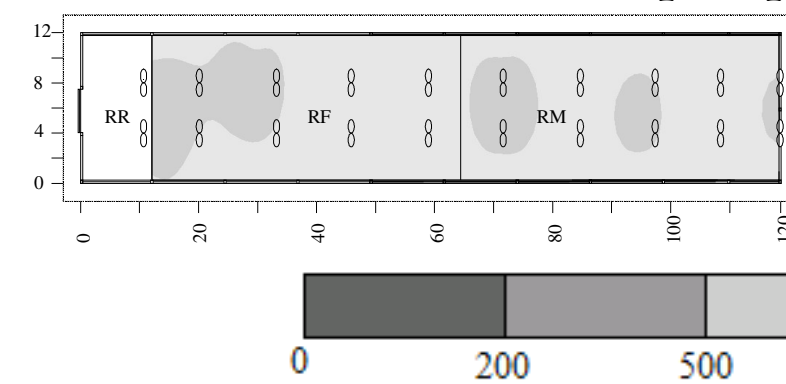

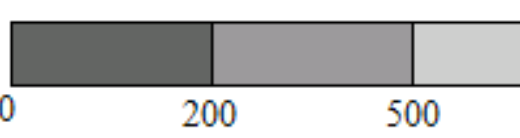

D.
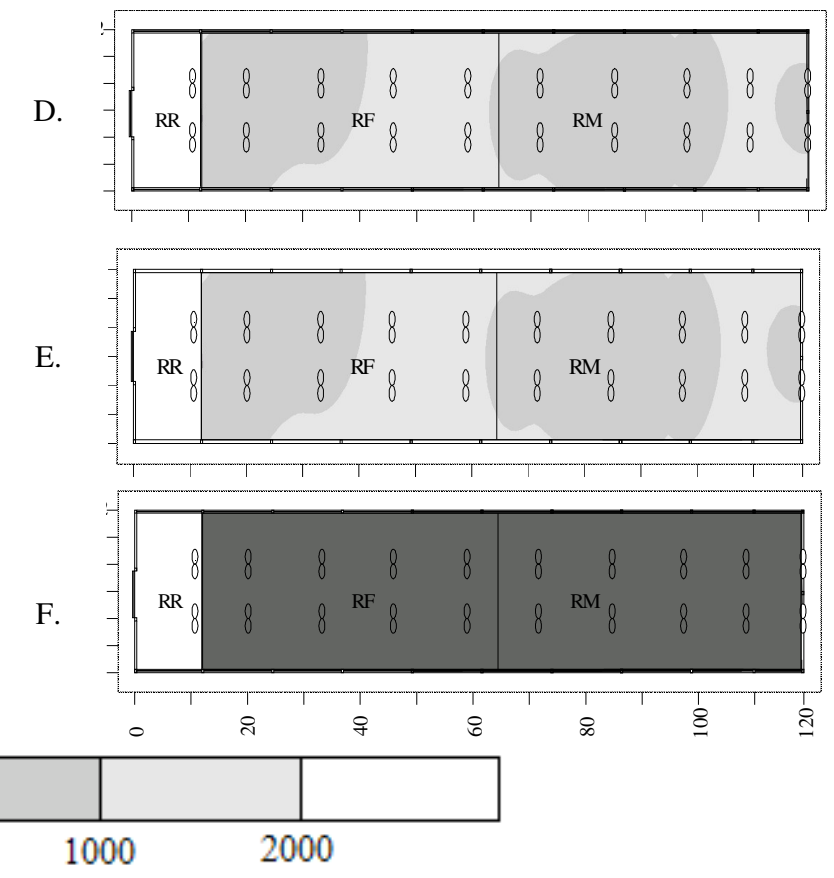

FIGURA 7. Distribuição espacial da iluminância, no interior de um galpão comercial para produção de frangos de corte, em diferentes horários ao longo do dia: A) 8h; B) 10h; C) 12h; D) $14 \mathrm{~h}$; E) $16 \mathrm{~h}$, e F) $18 \mathrm{~h}$. Spatial distribution of luminance, inside of a commercial broiler chicken house at different times throughout the day, A) 8:00, B) 10:00, C) 12:00, D) 14:00, E) 16:00, and F) 18:00. 


\section{CONCLUSÕES}

Com base nos resultados, pode-se concluir que os frangos de corte Hybro, machos e fêmeas, de forma geral, estiveram submetidos a condições ambientais inadequadas à criação nos horários mais quentes do dia, entre 12 e $16 \mathrm{~h}$.

O sistema de resfriamento evaporativo pode ser mais bem utilizado, uma vez que a UR se encontra entre 50 e $70 \%$ e assim proporciona melhores condições produtivas aos animais.

A espacialização das variáveis relacionadas ao ambiente produtivo proporciona a obtenção de informações mais detalhadas sobre o sistema estudado.

\section{AGRADECIMENTOS}

À CAPES, ao CNPq e à FAPEMIG pelo suporte financeiro a esta pesquisa.

\section{REFÊRENCIAS}

ABREU, P.G.; ABREU, V.M.N.; COLDEBELLA, A.; JAENISCH, F.R.F.; PAIVA, D.P. Condições térmicas ambientais e desempenho de aves criadas em aviários com e sem o uso de forro. Arquivo Brasileiro Medicina Veterinária e Zootecnia, Belo Horizonte, v.59, n.4, p.1.0141.020, 2007.

BARBOSA FILHO, J.A.D.; VIEIRA, F.M.C.; SILVA, I.J.O.; GARCIA, D.B.; SILVA, M.A.N.; FONSECA, B.H.F. Transporte de frangos: caracterização do microclima na carga durante o inverno. Revista Brasileira de Zootecnia, Viçosa-MG, v.38, n.12, p.2.442-2.446, 2009

BIAGGIONI, M.A.M.; MATTOS, J.M.; JASPER, S.P.; TARGA, L.A. Desempenho térmico de aviário de postura acondicionado naturalmente. Semina: Ciências Agrárias, Londrina, v.29, n.4, 961-972, 2008.

BUFFINGTON, D.E.; COLLASSO-AROCHO, A.; CANTON, G.H.; PIT, D. Black globe-humidity index (ITGU) as comfort equation for dairy cows. Transactions of the ASAE, St. Joseph, v.24, n.3, p.711-714, 1981.

CAMBARDELLA, C.A.; MOORMAN, T.B.; NOVAK, J.M.; PARKIN, T.B.; KARLEN, D.L.; TURVO, R.F.; KONOPA, A.E. Field-scale variability of soil properties in central Iowa soils. Soil Science Society of American Journal, Madison, v.58, n.5, p.1.501-1.511, 1994.

CRESSIE, A.G. Statistics for spatial data. New York: Wiley, 1993. 900 p.

DAMASCENO, F.A. Bem-estar do animal e do trabalhador em galpões avícolas climatizados. 2008. 195 f. Dissertação (Mestrado em Engenharia Agrícola) - Universidade Federal de Lavras, Lavras, 2008.

DIGGLE, P.J.; RIBEIRO JR, P.J. Model-based geostatistic. New York: Springer, 2006. 230 p.

DOWNS, K.M.; LIEN, R.J.; HESS, J.B.; BILGILI, S.F.; DOZIER III, W.A. The effects of photoperiod length, light intensity, and feed energy on growth responses and meat yield of broilers. Journal Applied Poultry Research, Stanford, v.15, n.3, p.406-416, 2006.

FARIA, F.F.; MOURA, D.J.; SOUZA, Z.M.; MATARAZZO, S.V. Variabilidade espacial do microclima de um galpão utilizado para confinamento de bovinos de leite. Ciência Rural, Santa Maria, v.38, n.9, p. 2.498-2.505, 2008.

FURTADO, D.A.; DANTAS, R.T.; NASCIMENTO, J.W.B.; SANTOS, J.T.; COSTA, F.G.P. Efeitos de diferentes sistemas de acondicionamento ambiente sobre o desempenho produtivo de frangos de corte. Revista Brasileira de Engenharia Agrícola e Ambiental, Campina Grande, v.10, n.2, p.484-489, 2006. 
GOOVAERTS, P. Geostatistical modeling of uncertainty in soil science. Geoderma, Amsterdam, v.103, n.1-2, p.3-36, 2001.

MCBRATNEY, A.G.; WEBSTER, A.G. Choosing functions for semi-variograms and fitting them to sampling estimates. Journal of Soil Science, Oxford, v.37, p.617-39, 1986.

MEDEIROS, C.M.; BAÊTA, F.C.; OLIVEIRA, R.F.M.; TINÔCO, I.F.F.; ALBINO, L F.T.A.; CECON, P.R. Efeitos da temperatura, umidade relativa e velocidade do ar em frangos de corte. Engenharia na Agricultura, Viçosa-MG, v.13, n.4, p.277-286, 2005.

MILES, D.M.; ROWE, D.E.; OWES, P.R. Winter broiler litter gases and nitrogen compounds: temporal and spatial trends. Atmospheric Environment, Amsterdam, v.42, n.14, p.3.351-3.363, 2008.

MIRAGLIOTTA, M.Y.; NÄÄS, I.A.; MANZIONE, R.L.; NASCIMENTO, F.F. Spatial analysis of stress conditions inside broiler house under tunnel ventilation. Sciencia Agricola, Piracicaba, v.63, n.5, p.426-432, 2006.

SANTOS, P.A.; YANAGI JÚNIOR, T.; TEIXEIRA, V.H.; FERREIRA, L. Ambiente térmico no interior de modelos de galpões avícolas em escala reduzida com ventilação natural e artificial dos telhados. Engenharia Agrícola, Jaboticabal, v.25, n.3, p.575-584, 2005.

SOUZA, S.R.L.; NÄÄS, I.A.; MARCHETO, F.G.; SALGADO, D.D. Análise das condições ambientais em sistemas de alojamento "freestall" para bovinos de leite. Revista Brasileira de Engenharia Agrícola e Ambiental, Campina Grande, v.8, n.2-3, p.299-303, 2004.

VIEIRA, S.R. Geoestatística em estudos de variabilidade espacial do solo. In: NOVAIS, R.F. et al. (Eds.). Tópicos em ciência do solo. Viçosa-MG: Sociedade Brasileira de Ciência do Solo, 2000. v.1, p.1-53. 